\title{
Considerações sobre o Fomento de Pesquisa em Psicologia do Desenvolvimento Humano no Brasil: História e Desafios
}

Consideration about the Furtherance of Research in Psychology of Human Development in Brazil: History and Challenges

Consideraciones sobre el Fomento de Pesquisa en Psicología del Desarrollo Humano en el Brasil: Historia y Desafíos

André de Carvalho-Barreto,

Pollianna Galvão Soares \&

Silviane Bonaccorsi Barbato

Universidade de Brasília

http://dx.doi.org/10.1590/1982 - 370000772013

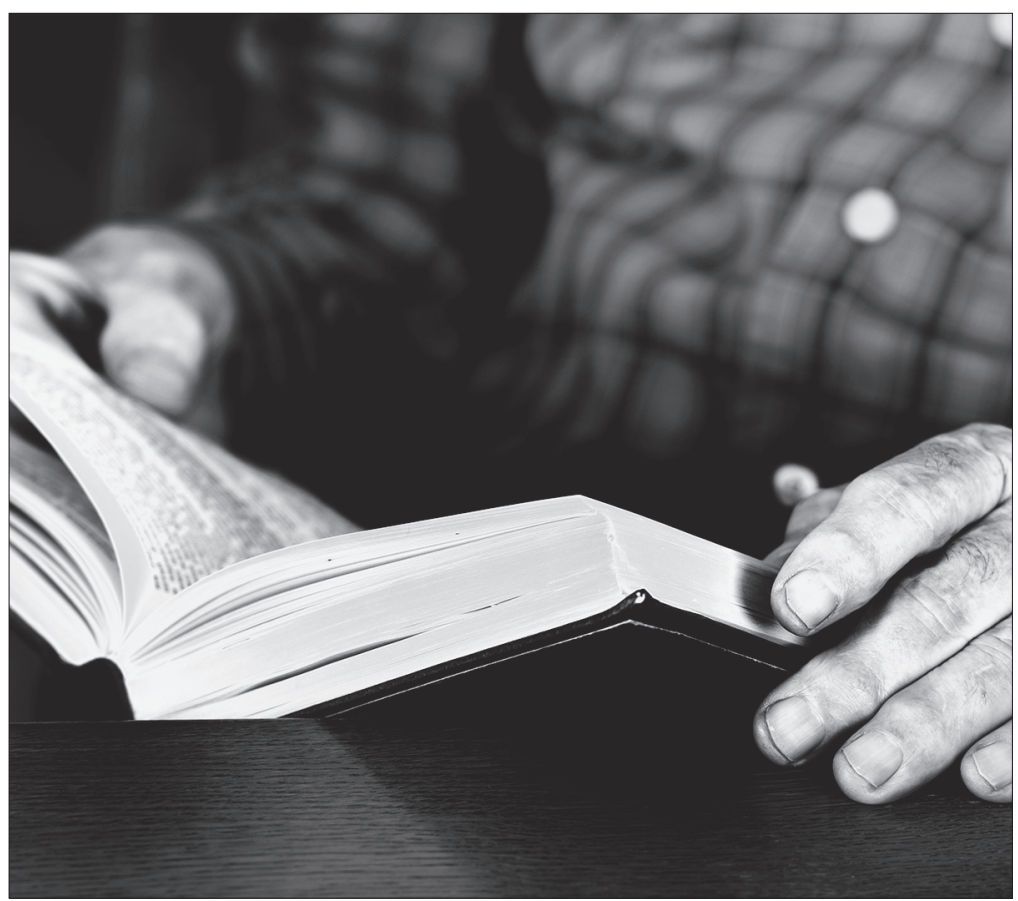


Resumo: O presente trabalho objetiva discutir o fomento à pesquisa no Brasil, destacando os papéis da história e das políticas de pesquisa e desenvolvimento científico, assim como das agências de pesquisa e associações relacionadas à área da Psicologia do Desenvolvimento Humano. Inicialmente, foi realizado um resumo da história da ciência brasileira e sua aplicabilidade no desenvolvimento humano. Posteriormente, é discutido como a Ciência do Desenvolvimento é interdisciplinar e a importância do aprofundamento do diálogo da Psicologia com outras áreas de conhecimento para a criação de novas tecnologias. São apresentadas, finalmente, possíveis tendências e mudanças científicas para a implementação de novos campos de conhecimento, considerando a Psicologia do Desenvolvimento Humano como área relevante para essas mudanças. Conclui-se que os recursos, assim como as pesquisas, estão inseridos em contextos históricos, sociais, culturais e econômicos dinâmicos, que devem ser considerados na captação de recursos.

Palavras-chave: Psicologia do desenvolvimento. Fomento à pesquisa. Agências de fomento.

Abstract: This paper aims at discussing furtherance of research in Brazil, highlighting the roles of research history and policies and scientific development, as well as research agencies and associations related to the Psychology of Human Development field. Initially, an overview of the Brazilian sciences history and it applicability in human development was carried out. Later, one discusses how interdisciplinary is the science of development and the importance of deepening the dialogue of Psychology with other areas of knowledge in order to set new techniques. Finally, possible scientific trends and changes to implement new fields of knowledge are presented, considering the Psychology of Human Development as relevant area for those changes. One concludes that resources, as well as researches, are inserted in dynamic historical, social, cultural, and economic contexts that should be considered when fundraising.

Keywords: Developmental psychology. furtherance of research, develoopment agencies.

Resumen: El presente trabajo objetiva discutir el fomento a la pesquisa en el Brasil, destacando los papeles de la historia y de las políticas de pesquisa y desarrollo científico, así como de las agencias de pesquisa y asociaciones relacionadas al área de la Psicología del Desarrollo Humano. Inicialmente fue realizado un resumen de la historia de la ciencia brasileña y su aplicabilidad en el desarrollo humano. Posteriormente, es discutido cómo la ciencia del desarrollo es interdisciplinaria y la importancia de la profundización del diálogo de la Psicología con otras áreas de conocimiento para la creación de nuevas tecnologías. Son presentadas, finalmente, posibles tendencias y mudanzas científicas para la implementación de nuevos campos de conocimiento, considerando la Psicología del Desarrollo Humano como área relevante para esas mudanzas. Se concluye que los recursos, así como las pesquisas, están inseridos en contextos históricos, sociales, culturales y económicos dinámicos, que deben ser considerados en la captación de recursos.

Palabras-clave: Psicología del desarrollo. fomento a la pesquisa, agencias de fomento. 
O presente trabalho objetiva discutir o fomento à pesquisa no Brasil, especialmente, às pesquisas em Psicologia e na área da Ciência do Desenvolvimento, destacando agências financiadoras e associações. Com o avanço da tecnologia a partir do início do século XX e a "era do desenvolvimento científico" (Pires-Alves, Paiva, \& Falleiros, 2010, p. 156) instaurada após a Segunda Guerra, foram criados diversos organismos intergovernamentais, governamentais e não governamentais destinados a promover o avanço científico e a colaboração entre países.

O fim da década de 1940 e início da de 1950 são caracterizadas por um expressivo avanço em políticas científicas e de gestão governamental no Brasil. Em 1948, um grupo de professores pesquisadores que decidiu que o Brasil precisava ter mais ciência, sendo necessária uma sociedade que promovesse o desenvolvimento da ciência nacional, propõe a Sociedade Brasileira para o Progresso da Ciência (SBPC). Para acompanhar as mudanças científicas mundial e regionais, em 1951, o governo federal apoia essa necessidade e constitui o Conselho Nacional de Pesquisa (CNPq) - que em 1974 mudou seu nome para Conselho Nacional de Desenvolvimento Científico e Tecnológico - e a Campanha Nacional de Aperfeiçoamento de Pessoal de Nível Superior (atual Capes - Coordenação de Aperfeiçoamento de Pessoal de Nível Superior). Universidades federais e estaduais foram implementadas também nessa década nos estados com incentivo federal e apoio estrangeiro (Capes, 2010; CNPq, 2010; SBPC, 2011).

O CNPq, no início de sua fundação, passa a oferecer mais bolsas às ciências relacionadas à Física Nuclear - por interesse em produzir esse tipo de tecnologia - e às Ciências Biológicas, por elas já serem bastante desenvolvidas no Brasil. Como nesse período o país teve expressivo aumento da produção indus- trial, paralelamente à baixa infraestrutura da administração pública, a Capes surgiu para incentivar a formação profissional, fomentando pesquisas que visassem ao aumento da produtividade industrial e provessem a manutenção ou reorientação das políticas públicas que atendessem às demandas da sociedade e das universidades que estavam sendo implementadas. A situação de centralização de fomento em algumas áreas se agravou no fim da década de 1950, com a criação da Comissão Nacional de Energia Nuclear (CNEN), ocasionando a transferência de mais da metade dos recursos das outras agências de fomento (Capes, 2010; CNPq, 2010).

Observou-se, então, já nas décadas de 1950 e 1960, que áreas de conhecimento consideradas pelos governos federal e estaduais como menos estratégicas, a exemplo da Psicologia do Desenvolvimento, que no período era voltada, especialmente, para o desenvolvimento infantojuvenil, recebia menos fomento que a Física, Engenharias e Ciências Biológicas e da Saúde (CNPq, 2010).

Dois anos após a criação da Capes e do CNPq, os Ministérios da Saúde e da Educação são consolidados como entidades distintas. Na década seguinte, 1960, alguns dos estados mais ricos da federação (e.g., São Paulo e Rio Grande do Sul) constituíram as primeiras Fundações de Apoio à Pesquisa nos estados, inserindo-se no desenvolvimento científico nacional (FAPESP, 2010). Essa política de Fundações de Apoio à Pesquisa nos estados prossegue pelas décadas seguintes passando a existir em 17 estados mais o Distrito Federal até o fim da década de 1990.

Nas décadas de 1950 e 1960 ainda, Capes e CNPq implementaram financiamento para pesquisadores que desejassem realizar seus cursos de pós-graduação no exterior. Esses recém-doutores que regressaram ao país au- 
xiliaram a implantar os primeiros programas de pós-graduação. Outros, no entanto, por maior incentivo para suas pesquisas nos países estrangeiros e melhor condição de vida socioeconômica, optaram por não regressar (CNPq, 2010). Com o aumento expressivo de pesquisas em 1985, foi criado o Ministério da Ciência e Tecnologia (MCT), pelo qual o CNPq passa a responder. Para regularizar a nova demanda de programas de pós-graduação que surgia, a Capes, na década de 1990, passou por uma reestruturação para diversificar suas atividades, assumindo também a atribuição de avaliadora desses programas. No final da década de 1990, o MCT criou o primeiro Fundo Setorial (CT-Petro), objetivando com esse tipo de fundo fomentar áreas estratégicas de desenvolvimento no país por recursos de instituições públicas e privadas (Capes, 2010).

$\mathrm{Na}$ Psicologia, os dois primeiros programas de mestrado foram instituídos na Universidade de São Paulo (USP), em 1970, direcionados para a Psicologia Experimental e para a Psicologia Escolar e do Desenvolvimento Humano, sendo igualmente esses dois programas os primeiros a implementar, em 1974, o doutorado em Psicologia nessas áreas (Carvalho, 1999; Yamamoto, 2006). Nessa mesma década, em 1971, também pela USP, é fundada a Sociedade Brasileira de Psicologia (SBP), que tinha entre os seus objetivos principais promover a Psicologia como ciência, realizando, assim, em outubro daquele ano, o primeiro evento científico nacional da área (Gorayeb, 1990).

Objetivando congregar os programas de pósgraduação para fomentar e estimular a formação de profissionais para pesquisa e pós-graduação em Psicologia, em 1983, foi fundada a Associação Nacional de Pesquisa e Pós-Graduação em Psicologia (ANPEPP), financiada, inicialmente, pelo CNPq. Basicamente, essa Associação organiza seus asso- ciados em Grupos de Trabalhos (GTs) com linhas específicas de investigação na Psicologia. Nos GTs, são traçadas estratégias de publicação, consolidação das linhas, intercâmbio de informações e estratégias de captação de recursos (ANPEPP, 2003).

Por interferência dessa Associação, a Psicologia, que ocupava até o início da década de 1980 a quinta posição de solicitantes de recursos financeiros na Área de Ciências Humanas e Sociais do CNPq, passou para o segundo lugar ainda naquela década (CNPq, 2010; Guedes, 2002). Os resultados do estudo de Seidl de Moura e Moncorvo (2006), em 2004, sobre os GTs da ANPEPP, identificaram que, aproximadamente, $40 \%$ deles tratam de temas ligados à Ciência do Desenvolvimento Humano, indicando essa disciplina como uma das que mais captavam recursos. Devido ao crescimento das pesquisas em Psicologia do Desenvolvimento, em 1998, um grupo de pesquisadores brasileiros funda a Associação Brasileira de Psicologia do Desenvolvimento (ABPD), que objetivava fomentar a pesquisa na área da Ciência do Desenvolvimento Humano, sobretudo, pela troca de informações sobre estudos entre pesquisadores (ABPD, 1998).

Dentre as metas de captação dos pesquisadores da Psicologia estão a inserção deles nas bolsas de Produtividade em Pesquisa (PQ) e a aprovação de seus projetos de pesquisa nos Editais Universal e das Ciências Humanas, Sociais e Sociais Aplicadas do CNPq. Esses editais, em suma, visam a atender às demandas de pesquisas que ofereçam uma contribuição significativa para o desenvolvimento científico e tecnológico nacional (Neves, Antunes, Júnior, \& Gorgati, 2007; Yamamoto, 2010), podendo esses fomentos serem submetidos e obtidos pessoalmente, ou seja, o pesquisador doutor pede diretamente o recurso para a agência - pedido "balcão" - ou por solicitação conjunta e articulada institucionalmente entre pesquisadores. 
As bolsas PQ do CNPq pretendem valorizar pesquisadores com elevada produção e inserção em suas disciplinas. Esse auxílio é concedido individualmente, levando em consideração o mérito da proposta e a adequação do pesquisador nos pré-requisitos delimitados pelo CNPq e pelos Comitês de Assessoramento de cada área. O valor das bolsas aumenta segundo o nível do pesquisador, que inicia no nível 2, seguindo para os níveis 1D, 1C, 1B e 1A (Neves, Antunes, Júnior, \& Gorgati, 2007; Yamamoto, 2010). Na área da Psicologia do Desenvolvimento constavam, em 2011, 59 pesquisadores com nível 2, 15 1D, sete 1C, 11 1B e nove $1 \mathrm{~A}$. Destes, 25 eram homens e 76 mulheres, 56 eram de instituições do Sudeste, 22 do Sul, 14 do Nordeste, nove do Centro-Oeste e três do Norte brasileiro (CNPq, 2011).

Apesar de a Psicologia do Desenvolvimento ser uma subárea da Psicologia, a distribuição dos bolsistas PQs por região do país nessa área coincide com as outras áreas de conhecimento, ou seja, o Sudeste é o detentor da maioria das bolsas, seguido pelo Sul, Nordeste, Centro-Oeste e Norte (Cavalcante et al., 2008; Martelli-Junior et al., 2010). Na distribuição de fomento entre instituições e pesquisadores pelo Edital Universal, a desigualdade entre regiões do Brasil é também presente (Yamamoto, 2000). Na distribuição entre as regiões brasileiras dos recursos do primeiro Edital Universal lançando em 2000, para a Psicologia, por exemplo, o Sudeste representou $63 \%$ da arrecadação, seguido pelo Sul, com 15\%; Nordeste, com 15\%; CentroOeste, com 14\%; e Norte, que não obteve arrecadação.

O levantamento apresentado divulga ainda que, dos recursos totais alocados para o Edital de 2000, somente $6 \%$ da demanda nacional foi atendida. Na Psicologia, dos 137 projetos encaminhados, apenas dez foram selecionados. Nessa arrecadação, três foram destinados para pesquisadores nível 1A, dois para nível 1B e dois nível 1C (Yamamoto, 2009).

Dados do resultado do Edital Universal de 2010, todavia, indicaram uma mudança expressiva dessa situação na Psicologia. Ao todo, 63 investigações psicológicas foram contempladas. Destas, dois autores eram pesquisadores $1 \mathrm{~A}$, quatro $1 \mathrm{~B}$, quatro $1 \mathrm{C}$, três 1D e 17 Nível 2. Dentre os projetos aprovados, 12 eram de pesquisadores direcionados à Ciência do Desenvolvimento Humano. Um dado, contudo, chama atenção: dos pesquisadores contemplados nesse Edital, sete não tinham doutorado, mestrado ou graduação em Psicologia. Outro dado relevante é que, apesar do aumento de projetos aprovados na Psicologia, muitos pesquisadores da área têm buscado recursos em outras áreas de concentração, como no Fundo Setorial da Saúde (CNPq, 2011). Ainda que tenha se observado o aumento na regularidade de editais e dos seus recursos na última década, eles ainda são insuficientes perante o fluxo de pesquisas em andamento.

\section{A Ciência do Desenvolvimento como área interdisciplinar}

A necessidade de se compreender os processos de desenvolvimento do ser humano é um tema antigo na ciência que desde muitos séculos vem intrigando filósofos e estudiosos de diversas disciplinas (Cairms, Elder, \& Costello, 1996). Os estudos sobre desenvolvimento humano na Psicologia tiveram seu período formativo entre os anos de 1882 e 1912 na França e nos Estados Unidos (Cairns, 1983). Nessa época, os focos de estudo eram a Psicobiologia, a Psicologia da Personalidade e o Desenvolvimento Cognitivo, tendo como principais pensadores Alfred Binet (1857-1911), William Preyers (1841-1897) e Stanley Hall (1844-1924). Ainda no período inicial dos estudos sobre o 
desenvolvimento humano, essa área já apresentava tecnologias relevantes capazes de auxiliar, como, por exemplo, na seleção de soldados que seguiriam para a frontaria de batalha durante a Primeira Guerra Mundial.

Estudiosos da história da Ciência do Desenvolvimento Humano na Psicologia dividem em quatro momentos os focos de estudo dessa ciência após seu período formativo (Biaggio \& Monteiro, 1998; Bronfenbrenner, 2011; Cairns, 1983; Mota, 2005). O primeiro deles ocorreu nas décadas de 1920 e 1930 , tendo como principais interesses de estudo o desenvolvimento intelectual, a maturação e o crescimento do ser humano centrado na criança. No segundo momento (1940-1959), por influência do contexto da Segunda Guerra Mundial, o objetivo de análise do período era, especialmente, o estudo de relações e correlações de variáveis que afetavam o desenvolvimento humano. Nesse período, as pesquisas enfatizavam a criação de tecnologias de intervenção. Seus conhecimentos passam a ser utilizados tanto com crianças e adolescentes no contexto escolar quanto no tratamento de ex-combatentes da Segunda Guerra Mundial.

O terceiro, compreendido entre 1960 e 1989, caracterizou-se pela emergência de novas pesquisas teóricas no campo do desenvolvimento humano, entre as quais se destacam o desenvolvimento infantil e da adolescência (Biaggio \& Monteiro, 1998; Cairns, 1983). Muitas das investigações foram organizadas em volta de modelos conhecidos como os três grandes sistemas: (a) Teoria do Desenvolvimento Cognitivo de Piaget; (b) Teoria Psicanalítica; (c) Teoria da Aprendizagem (Bronfenbrenner, 2011).

As transformações sociais e tecnológicas ocorridas no final do século XX suscitaram a necessidade de se construir um novo modelo de Ciência do Desenvolvimento com refle- xões sobre o ser humano e com enfoque tanto no fenômeno da experiência humana quanto nos seus processos, abrangendo alianças entre diferentes áreas - incluindo as tecnológicas aplicadas - em um esforço de abrangências inter e intradisciplinares. Esse novo modelo incentivava o diálogo da Psicologia com outras áreas como com a Genética, Medicina, Saúde Pública, Biologia, Economia, Engenharias, Ciências Sociais, Linguística, Comunicação, Artes, Educação e Filosofia. As relações indivíduo-cultura passaram a ser constituintes do sujeito e estabelecidas em práticas culturais concretizadas e atualizadas nas interações e atividades diversas em contextos históricos e sociais específicos. Esses contextos exercem significativa influência nas interações e atividades dos sujeitos que são alvo da investigação científica.

A emergência de sistemas heterogêneos globais instaurou novas tendências nas pesquisas com enfoque nas relações entre "zonas de contato entre culturas, a complexidade do self, identidade e a experiência de incertitude" (Hermans \& Kempen, 1998, p. 1.111). Essas ênfases derivam da relevância que se passou a atribuir aos diferentes aspectos de desenvolvimento local e dimensões da globalização para a compreensão do desenvolvimento humano, como, por exemplo, os processos de identificação, migração, hibridização (Canclini, 2003).

Os fatores externos e as relações estabelecidas entre as pessoas com a concretização de novas condições de socialização começaram a ser estudados em uma perspectiva sistêmica e a concepções de ser humano e de desenvolvimento, anteriormente calcada nas aptidões inatas e/ou no determinismo biológico, foi repensada (Vasconcelos, 2002). Foi nesse cenário, portanto, que emergiu um novo paradigma da Ciência do Desenvolvimento Humano inter e multidisciplinar, pelo qual se busca não apenas a compreensão do 
desenvolvimento como um fenômeno complexo, mas procura sistematizar o conhecimento envolvendo fatores históricos, contextuais e relacionais (Valsiner \& Connolly, 2003).

Assim, a partir das trocas e avanços em teorizações e as possibilidades de aplicação, estudos em desenvolvimento humano também passaram a ser fonte para diversas áreas científicas e tecnológicas ao longo das últimas décadas, havendo ainda espaços a serem conquistados. Ao serem considerados os quatro momentos da história da Ciência do Desenvolvimento, é destacado a relevância dessa ciência para apoio na construção de conhecimentos teóricos e empíricos sobre o desenvolvimento da pessoa nessas novas condições de socialização nos diferentes contextos de nossa sociedade. Os resultados de estudos nos três primeiros momentos da história sobre o desenvolvimento infantojuvenil, nos diversos paradigmas e perspectivas, foram aplicados em pesquisa básica e aplicada com enfoque em migração, políticas públicas, apoio às tecnologias na educação, no trabalho, na saúde, na assistência, dentre outras áreas.

O período que compreendeu a década final do século XX até a primeira década do século XXI destaca-se como o momento de maior mudança dessa ciência, ocorrida por forte influência de pensadores da Psicologia do Desenvolvimento - Baltes, Bronfenbrenner, Elder, Hinde, Lewin, Magnusson, Valsiner, Vygotsky, entre outros. O desenvolvimento do ser humano, nesse quarto momento, é estudado ao longo do curso de vida da pessoa, ou seja, compreendendo todas as fases e estágios do desenvolvimento humano, incluindo a tradicional ênfase na infância e adolescência (Bronfenbrenner, 2011; Mota, 2005).

A Psicologia do Desenvolvimento Humano, como uma das disciplinas da Ciência do De- senvolvimento, ressignifica e amplia a função de compreender o desenvolvimento em períodos específicos, abrangendo todas as fases do desenvolvimento continuamente, sob uma perspectiva histórica e processual. Além disso, os paradigmas atuais da Psicologia do Desenvolvimento orientam que se ultrapasse a análise do indivíduo, seguindo para a sociedade, a família e contemplando, ainda, aspectos culturais, históricos, políticos e econômicos (Dessen, 2005; Gergen, 2010; Mather, 2000; Morawski, 2005; Parker, 2009; Smedslund, 2009; Smith, 2005). Portanto, embora a Psicologia avance no sentido de contemplar mais aspectos na compreensão do seu objeto de estudo, passa a situá-lo em caráter interdisciplinar, uma vez que compreende as colaborações de sua atividade científica para a compreensão multifacetada do fenômeno humano.

\section{Tendências e desafios para o fomento da pesquisa em desenvolvimento humano}

Conciliar áreas de conhecimento que pouco dialogaram ao longo da história das ciências implica em desafios para a interdisciplinaridade (Teixeira, 2004; Richards, 2002). Os principais incluiriam a organização e coordenação de projetos de pesquisa interdisciplinar por envolver pesquisadores que, muitas vezes, pertencem a instituições diferentes e partem de perspectivas epistemológicas distintas, o que ocasionam diferenças de conceitos e compreensões sobre um mesmo fenômeno (Almeida, et al 2004). Por exemplo, nos diálogos possíveis entre Psicologia Desenvolvimento e Neurociências; Psicologia do Desenvolvimento, Sociologia e Antropologia; e entre Psicologia do Desenvolvimento, Psicologia da Aprendizagem e Ciências da Computação.

A prática interdisciplinar é, portanto, mais um ponto de partida, não sendo dada por 
regras preestabelecidas, fórmulas ou conceitos comuns (Teixeira, 2004). Um projeto interdisciplinar que envolva a Ciência do Desenvolvimento Humano é construído pelo respeito entre as áreas com uma dinâmica e pode gerar inovações que influenciam os fazeres além de avanços na compreensão dos fenômenos estudados. Inovações que impactam as práticas cotidianas societárias e o próprio fazer cientifico (Dessen, 2005).

Um exemplo pode ser ilustrado pela pesquisa de Santos (2010) realizada sobre transporte escolar rural, desenvolvida sob as perspectivas da Psicologia do Desenvolvimento Humano somada à Ciência Política. Esse estudo teve entre os seus objetivos investigar o impacto no desenvolvimento de crianças e adolescentes matriculados em escolas em áreas rurais com dificuldade de acessibilidade. Nesse estudo, como em outros que envolvem a interdisciplinaridade com área do desenvolvimento humano, fica evidenciado como o trabalho de pesquisa nessa área pode criar tecnologias essenciais para/com outras áreas e práticas organizacionais e institucionais (e.g., hospitalar, educacional, habitação, transporte, militar, dentre outras).

Esse é um dos exemplos pelos quais se pode afirmar que ações e políticas de apoio às vinculações entre pesquisadores de áreas diversas têm sido desencadeadas nesse século e, principalmente, entre instituições diferentes, dada a ênfase contemporânea da internacionalização entre os pesquisadores em Psicologia (Anpepp, 2012). Para facilitar a obtenção desse perfil interdisciplinar e de internacionalização das produções, vinculações entre pesquisadores de áreas diversas e, principalmente, entre instituições diferentes podem ratificar a proposta. Observa-se que as associações de pesquisa, como a SBPC, Anpepp, ABPD e SBP, assumiram uma função central no fortalecimento de linhas e projetos na Ciência do Desenvolvimento, entre outras, facilitando a captação de recursos para pesquisas de caráter interdisciplinar (e.g., Yamamoto \& Seidl de Moura, 2010). A possibilidade, portanto, da Ciência do Desenvolvimento de constituir investigações em áreas distintas da Psicologia, com a solidificação de redes interdisciplinares, eleva significativamente a viabilidade de obtenção de fomento em editais além do Universal e das Ciências Humanas, Sociais e Sociais Aplicadas do CNPq.

No entanto, deve ser clarificada a relação dialética existente na interdisciplinaridade (Teixeira, 2004). Da mesma forma que a Psicologia do Desenvolvimento precisa dialogar com outras áreas como as Engenharias, Ciências Biológicas, Ciências da Saúde, na perspectiva da Ciência do Desenvolvimento, essas áreas também necessitam buscar a complementaridade entre si. Afirma-se que a Psicologia do Desenvolvimento tem contribuições potenciais para a produção de pesquisas robustas e amplas na Ciência do Desenvolvimento, a fim de somar força ao empreendimento científico na sua coletividade visando, assim, a consolidar tecnologias eficazes às necessidades sociais, públicas e econômicas do país (Parker, 2009).

Outro desafio diz respeito à internacionalização da produção científica do país. Apesar de pesquisadores doutores com nível de produção acadêmica elevada apresentarem maior probabilidade de obtenção de recursos para suas investigações considerando-se os parâmetros das agências de fomento (CNPq, 2010; MCT, 2011) e as Ciências Humanas serem as que possuem mais doutores, esses pesquisadores tem direcionado, aproximadamente, $85 \%$ de suas produções para periódicos nacionais, diferentemente das Ciências Exatas e da Terra, Biológicas e da Saúde, Engenharia e Computação, que destinam, no geral, uma média de $65 \%$ de suas 
produções para periódicos internacionais (Alencar, 2006).

As instituições de fomento, ao longo dos anos, têm proposto uma distribuição piramidal de recursos, ou seja, editais com vários níveis de solicitação de auxílio à pesquisa têm optado por atender mais às demandas dos projetos de menor custo em detrimento dos mais elevados. Assim, os recursos são distribuídos para uma quantidade maior de pesquisadores com o valor mínimo de fomento e um número limitado para investigadores com o valor máximo de apoio à pesquisa (CNPq, 2010).

A pesquisa no Brasil em sua história segue o fluxo dos interesses nacionais e mundiais (Pires-Alves, Paiva, \& Falleiros, 2010). O progresso socioeconômico nacional, as tendências de pesquisa mundiais e, especialmente, as demandas macrossistêmicas que surgem no Brasil, possibilitam uma análise sobre os direcionamentos de fomento. Nesse contexto, ao considerarmos a produção do conhecimento e de teorias sobre o processo de desenvolvimento, necessitamos abarcar questões que fundamentam a identificação de novos temas teóricos, empíricos e aplicados que enfoquem as problemáticas públicas, econômicas e tecnológicas prioritárias na política atual.

\section{Considerações Finais}

Projetos relevantes de investigação no desenvolvimento humano são necessários, sendo importante considerar que os recursos, assim como as pesquisas, estão inseridos em contextos históricos, sociais, culturais, econômi$\cos$ e, especialmente, dinâmicos. Nessa direção, enfatiza-se a relevância de a Psicologia possibilitar articulações interdisciplinares com outros campos do saber, especialmente com vistas ao aumento da qualidade de vida das pessoas nos diversos contextos atuais de nossa sociedade. Assim, a Psicologia e a Ciência do Desenvolvimento devem adotar estratégias político-institucionais que visem a direcionar as demandas advindas das agências de fomento que tratem sobre questões relativas ao homem.

Dessa forma, unindo-se a outras áreas, a Psicologia e a Ciência do Desenvolvimento humano têm potencialidades não apenas para participar ativamente nas novas políticas de pesquisas, mas também mobilizar ações profissionais que liderem debates críticos sobre as tendências do fomento em pesquisa no país. Chama-se a atenção aos grupos de pesquisas em Psicologia para um necessário apoio mútuo entre as instituições, e mesmo entre as áreas, especialmente em relação às demandas de estudos específicos e transversais que surgem como tendência ao fomento em pesquisa da Ciência do Desenvolvimento. Os estudos que já têm reconhecimento nacional e que se enunciam relacionados às mudanças para a construção de novas agencialidades e instrumentos mediacionais, visando a serem insumos empíricos para atuações de prevenção e superação em diferentes frentes ambientalistas, organizacionais, comunicacionais e interculturais, são exemplos desse avanço ao qual precisamos persistir. 


\section{André de Carvalho-Barreto}

Doutorando em Processos do Desenvolvimento Humano e Saúde pela Universidade de Brasília, Brasília - DF. Brasil.

E-mail: andrecarvalhobarreto@yahoo.com.br

\section{Pollianna Galvão Soares}

Doutora em Processos de Desenvolvimento Humano e Saúde pela Universidade de Brasília, Brasília - DF. Brasil.

E-mail: polliannagalvao@yahoo.com.br

\section{Silviane Bonaccorsi Barbato}

Doutora em Processos de Desenvolvimento Humano e Saúde pela Universidade de Brasília, Brasília - DF. Brasil. Docente da Universidade de Brasília, Brasília - DF. Brasil.

\section{Endereço para envio de correspondência:}

Universidade de Brasília, Campus Universitário Darcy Ribeiro, Instituto Central de Ciências Sul, Instituto de Psicologia, Laboratório de Desenvolvimento Familiar. Asa Norte. CEP: 70904-970. Brasília - DF. Brasil.

Recebido 23/04/2013, 1ㅍ- Reformulação: 06/03/2014, Aprovado 26/08/2014. 
Alencar, H. (2006). Ciências Exatas e da Terra. In F. Sobral (Org.), Cadernos da SBPC - Ciência e tecnologia no Brasil (pp. 41-62). São Paulo: Sociedade Brasileira para o Progresso da Ciência.

Almeida, J., Gerhardt, T. E., Miguel, L. A., Netto, C. G. A. M., Verdum, R., Beck, F. L., \& Zanoni, M. (2004). Pesquisa interdisciplinar na pósgraduação: (Des)caminhos de uma experiência em andamento. Revista Brasileira de Pós-Graduacão, 1(2), 116-140.

Associação Brasileira de Psicologia do Desenvolvimento-ABPD. (1998). Ata de criação da Sociedade Brasileira de Psicologia do Desenvolvimento. Recuperado de: http://www.msmidia.com/sbpd/atacria.htm.

Associação Nacional de Pesquisa e Pós-Graduação em Psicologia-ANPEPP. (2003). Estatuto da ANPEPP [versão em PDF]. Recuperado de: http://www.anpepp.org.br/.

Biaggio, Â., \& Monteiro, J. K. (1998). A psicologia do desenvolvimento no Brasil e no mundo. In M. L. S. Moura, J. Correa, \& A. Spinillo (Orgs.), Pesquisas brasileiras em psicologia do desenvolvimento (pp. 15-31). Rio de Janeiro: Eduerj.

Bronfenbrenner, U. (2011). Bioecologia do desenvolvimento humano: tornando os seres humanos mais humanos (A. Carvalho-Barreto, Trad.). Porto Alegre: Artmed.

Cairms, R. B., Elder, G. H., \& Costello, E. J. (Org.) (1996). Developmental science. New York: Cambridge University.

Cairns, R. B. (1983). The emergency of developmental psychology. In P. H. Mussen (Ed.), Handbook of Child Psychology (pp. 41-101). New York: John Wiley.

Canclini, N. (2003). Culturas híbridas. São Paulo: Edusp.

Carvalho, A. M. A. (1999). Onde estão e o que fazem nossos mestres e doutores? Uma reflexão sobre critérios de avaliação de pós-graduação. Psicologia: Ciência e Profissão, 19(2), 54-63. doi: 10.1590/S141498931999000200008

Cavalcante, R. A. et. al. (2008). Perfil dos pesquisadores da área de odontologia no Conselho Nacional de Desenvolvimento Científico e Tecnológico (CNPq). Revista Brasileira de Epi- demiologia, 11(1), 106-113. doi: 10.1590/S1415-790X2008000100010

Conselho Nacional de Desenvolvimento Científico e Tecnológico - CNPq. (2010). Centro de memória do CNPq. Recuperado de: http://centrodememoria.cnpq.br/.

Conselho Nacional de Desenvolvimento Científico e Tecnológico CNPq. (2011). Plataforma Lattes. Recuperado de http://lattes.cnpq.br/.

Coordenação de Aperfeiçoamento de Pessoal de Nível Superior - CAPES. (2010). Página principal. Recuperado de: http://www.capes.gov.br/.

Dessen, M. A. (2005). Construindo uma ciência do desenvolvimento: passado, presente e futuro. In M. A. Dessen, \& A. L. Costa Júnior (Orgs.), A ciência do desenvolvimento humano: tendências atuais e perspectivas futuras (pp. 264-276). Porto Alegre: Artmed

Fundação de Amparo à Pesquisa do Estado de São Paulo. (2010). A FAPESP. Recuperado de: http://www.fapesp.br/.

Gergen, K. J. (2010). The acculturated brain. Theory \& Psychology, 20(6), 795-816. doi: 10.1177/0959354310370906

Gorayeb, R. (1990). Histórico da Sociedade Brasileira de Psicologia. Recuperado de: http://www.sbponline.org.br/historico.php/

Guedes, M. C. (2002). Para uma história da ANPEPP. Recuperado de: http://www.anpepp.org.br/historico.html\#Par a uma história da ANPEPP/.

Hermans, H. J. M., \& Kempen, H. J. G. (1998). Moving cultures: The perilous problems of cultural dichotomies in a globalizing society. American Psychologist, 53(10), 1111-1120. doi: . http://dx.doi.org/10.1037/0003066X.53.10.1111

Martelli-Junior, H. et al.. (2010). Pesquisadores do CNPq na área de medicina: comparação das áreas de atuação. Revista da Associação Médica Brasileira, 56(4), 478-483. doi: 10.1590/S0104-42302010000400024

Mather, R. (2000). The foundations of critical psychology. History of the Human Sciences, 13 (2), 85-100.

doi: 10.1177/09526950022120683 
Ministério da Ciência e Tecnologia-MCT. (2011). Fontes de financiamento. Recuperado de: http://www.mct.gov.br/

Mota, E. M. (2005) Psicologia do desenvolvimento: uma perspectiva histórica. Temas em Psicologia, 13(2), 105-111. Recuperado de http://pepsic.bvsalud.org/scielo.php?pid=S14 13-389X2005000200003\&script=sci_arttext

Morawski, J. G. (2005). Reflexivity and the psychologist. History of the Human Sciences, 18 (4), 77-105. doi: 10.1177/0952695105058472

Neves, M. A. S. P., Antunes, R. C., Junior, E. S. R., \& Gorgati, C. Q. (2007). Evolução das bolsas de produtividade em pesquisa e dos editais universais do CNPq no Programa Básico de Zootecnia: 2002 a 2006. Revista Brasileira de Zootecnia, 36(Supl.), 369-376. doi: 10.1590/S1516-35982007001000033

Parker, I. (2009). Critical psychology and revolutionary. Theory \& Psychology, 19(01), 71-92. doi: 10.1177/0959354308101420

Pires-Alves, F. A., Paiva, C. H. A., \& Falleiros, I. (2010). Saúde e desenvolvimento: a agenda do Pós-Guerra. In C. F. Ponte \& I. Falleiros (Orgs.), Na corda bamba de sombrinha: a saúde no fio da história (pp. 153-165). Rio de Janeiro: Fiocruz.

Richards, G. (2002). The psychology of psychology: A history grounded sketch. Theory \& Psychology, 12(1), 7-36. doi: 10.1177/0959354302121002

Santos, T. M. M. (2010). Transporte escolar rural na perspectiva de gestores e planejadores/operadores: subsídios para políticas públicas. Dissertação de Mestrado, Universidade de Brasília, Brasília, DF.

Seidl de Moura, M. L., \& Moncorvo, M. C. R. (2006). A psicologia do desenvolvimento no Brasil: tendências e perspectivas. In D. Colinvaux, L. B. Leite, \& D. D. Dell'aglio (Orgs.), Psicologia do desenvolvimento: reflexões e práticas atuais (pp. 115-132). São Paulo: Casa do Psicólogo.
Smedslund, J. (2009). The mismatch between current research methods and the nature of psychological phenomena. Theory \& Psychology, 19(6), 778-794. doi: 10.1177/0959354309345648

Smith, R. (2005). Does reflexivity separate the human sciences from the natural sciences? History of the Human Sciences, 18(4), 1-25. doi: 10.1177/0952695105058468

Sociedade Brasileira para o Progresso da Ciência - SBPC (2011). Página principal. Recuperado de: http://www.sbpcnet.org.br/.

Teixeira, O. A. (2004). Interdisciplinaridade: problemas e desafios. Revista Brasileira de PósGraduacão, 1(2), 57-69.

Valsiner, J., \& Connolly, K. (Orgs.) (2003). Handbook of developmental psychology. London: Sage.

Vasconcelos, M. J. E. (2002). Pensamento sistêmico: o novo paradigma da ciência. Campinas, SP: Papirus.

Yamamoto, O. H. (2000). Financiamento da pesquisa no Brasil: distorções e desigualdades. Estudos de Psicologia (Natal), 5(2), 279-287. doi: 10.1590/s1413-294×2000000200001

Yamamoto, O. H. (2006). Graduação e Pós-Graduação em Psicologia: Relações possíveis. Revista Brasileira de Pós-Graduacão, 3(6), 270-281.

Yamamoto, O. H. (2010). Pesquisa na Psicologia: reflexões sobre a formação. In Associação Nacional de Pesquisa e Pós-Graduação em Psicologia (Org.), XIII Simpósio de Pesquisa e Intercâmbio Científico em Psicologia. Anais Pesquisa em Psicologia: formação, produção e intervenção (pp. 183-195). Fortaleza, CE: Autor.

Yamamoto, M. E., \& Seidl de Moura, M. L. (2010) A psicologia evolucionista no Brasil. Estudos de Psicologia (Natal), 15(1), 53-54. doi: 10.1590/S1413-294X2010000100007 